\title{
The absolute rigidity of the Neveu-Schwarz and Ramond superalgebras
}

\author{
N. W. van den Hijligenberg ${ }^{\text {a) }}$ \\ Centre for Mathematics and Computer Science, P.O. Box 94079, 1090 GB Amsterdam, \\ The Netherlands \\ Yu. Yu. Kotchetkov \\ Institute of New Technologies in Education, Moscow, Russia
}

(Received 22 March 1996; accepted for publication 8 May 1996)

\begin{abstract}
We prove that the second cohomology group with coefficients in the adjoint module of both the infinite-dimensional Lie superalgebras $k(1)$ and $k^{+}(1)$, as well as their unique central extensions, which are known as the Neveu-Schwarz and the Ramond superalgebras, respectively, is equal to zero. This particularly implies that all these Lie superalgebras are rigid, i.e. they can only be deformed in a trivial manner. (C) 1996 American Institute of Physics. [S0022-2488(96)03809-1]
\end{abstract}

\section{INTRODUCTION}

From the general theory on deformations of algebraic structures (see, e.g., Ref. 1), we learn that constructions of deformations involve cohomology computations. The infinitesimal part of an algebra deformation can be represented as an element of the second cohomology group with coefficients in the adjoint representation. An algebra is called rigid if all its deformations are trivial, i.e. any algebra that is obtained from it by deformation is isomorphic to the original algebra by means of an isomorphism that depends on the deformation parameter, which reduces to the identity mapping when this deformation parameter is set equal to zero. A sufficient condition for the rigidity of an algebra is that its second cohomology group is equal to zero. Therefore, algebras that satisfy this condition are usually called absolutely rigid.

In recent years quite a number of papers have been written on the topic of deformations of nilpotent subalgebras of graded infinite-dimensional Lie algebras (see, e.g., Refs. 2-6). These works show that the nilpotent subalgebras have several nontrivial deformations that have a fairly simple algebraic description. In Refs. 7 and 8 the author shows that the situation is similar in the case of graded infinite-dimensional Lie superalgebras. With respect to the corresponding graded Lie (super)algebras, one could say there is common consent that these Lie (super)algebras are rigid. In this paper we show that the results of the cohomology computations for the positive nilpotent part of the Neveu-Schwarz and the Ramond Lie superalgebras presented in Ref. 7, can be fruitfully used to prove that these Lie superalgebras are absolutely rigid. In fact, the presented method of rigidity proving is another illustration of the usefulness of studying these nilpotent subalgebras.

The Neveu-Schwarz and Ramond superalgebras are usually called super-Virasoro algebras since they can be viewed as superanalogs of the Virasoro algebra. As the Virasoro algebra arises as the unique (up to equivalence) central extension of the Witt algebra, they are the unique central extensions of the simple Lie superalgebras $k(1)$ and $k^{+}(1)$, respectively (see, e.g., Ref. 9). Both superalgebras appear in mathematical physics (see, e.g., Refs. 10 and 11). For instance, in superstring theory (see, e.g., Ref. 12) the Neveu-Schwarz algebra describes the super-Virasoro algebra in the bosonic sector, whereas the Ramond algebra describes the super-Virasoro algebra in the fermionic sector.

${ }^{a)}$ Electronic mail: nico@cwi.nl 
The Neveu-Schwarz algebra can be presented as the $\mathbb{Z}_{2}$-graded vector space with $\left\{L_{p}, D\right\}_{p \in Z}$ as a basis of the even homogeneous part and $\left\{G_{q}\right\}_{q+1 / 2 \in Z}$ as a basis of the odd homogeneous part. Its commutator is defined by

$$
\begin{gathered}
{\left[L_{p}, L_{q}\right]=(p-q) L_{p+q}+\frac{1}{8}\left(p^{3}-p\right) \delta_{p+q, 0} D,} \\
{\left[L_{p}, G_{q}\right]=\left(\frac{1}{2} p-q\right) G_{p+q},} \\
{\left[G_{p}, G_{q}\right]=2 L_{p+q}+\frac{1}{2}\left(p^{2}-\frac{1}{4}\right) \delta_{p+q, 0} D,}
\end{gathered}
$$

and the property that $D$ is a central element. The Ramond algebra has $\left\{L_{p}, D\right\}_{p \in Z}$ as a basis of the even homogeneous part and $\left\{F_{q}\right\}_{q \in Z}$ as a basis of the odd homogeneous part. It is equipped with the commutator

$$
\begin{gathered}
{\left[L_{p}, L_{q}\right]=(p-q) L_{p+q}+\frac{1}{8} p^{3} \delta_{p+q, 0} D,} \\
{\left[L_{p}, F_{q}\right]=\left(\frac{1}{2} p-q\right) F_{p+q},} \\
{\left[F_{p}, F_{q}\right]=2 L_{p+q}+\frac{1}{2} p^{2} \delta_{p+q, 0} D,}
\end{gathered}
$$

where $D$ is defined to be central.

\section{THE RIGIDITY OF $k(1)$ AND NS}

The Lie superalgebra $k(1)$ (see Ref. 9) is defined as the $\mathbb{Z}_{2}$-graded vector space $\mathbb{C}\left[t, t^{-1}, \theta\right]$ equipped with the commutator

$$
[f, g]=\left(2 f-\theta \frac{\partial f}{\partial \theta}\right) \frac{\partial g}{\partial t}-\frac{\partial f}{\partial t}\left(2 g-\theta \frac{\partial g}{\partial \theta}\right)-(-1)^{|f|} \frac{\partial f}{\partial \theta} \frac{\partial g}{\partial \theta}, \quad f, g \in \mathbb{C}\left[t, t^{-1}, \theta\right] .
$$

The parameter $t$ is even and the parameter $\theta$ is odd. The symbol $|f|$ denotes the parity of the element $f$ in $\mathbb{C}\left[t, t^{-1}, \theta\right]$. As basis of $k(1)$ we choose $\left\{e_{p}\right\}_{p \in \mathbb{Z}}$ where

$$
e_{2 p}=t^{p+1}, \quad e_{2 p-1}=\theta t^{p} \quad(p \in \mathbb{Z}) .
$$

Note that the elements in this basis are homogeneous, in particular, the parity of $e_{p}$ equals $p$ modulo 2. The unique central extension of $k(1)$, which we denote by $N S$, is determined by the 2-cocycle $c \in Z^{2}(k(1))$, defined as

$$
c(f \wedge g)=\operatorname{Res}\left\{\left(\frac{\partial f}{\partial \theta}-\theta \frac{\partial f}{\partial t}\right) \frac{\partial^{2} g}{\partial t^{2}}-(-1)^{|f|} \frac{\partial^{2} f}{\partial t^{2}}\left(\frac{\partial g}{\partial \theta}-\theta \frac{\partial g}{\partial t}\right)\right\} .
$$

In this formula the symbol Res denotes the residue, i.e., $\operatorname{Res}(h)$ is the coefficient of the term $\theta t^{-1}$ in $h \in \mathbb{C}\left[t, t^{-1}, \theta\right]$. The wedge $(\wedge)$ in formula (5) denotes the $\mathbb{Z}_{2}$-graded antisymmetric tensor product, so for homogeneous elements $f$ and $g$ in $k(1)$ we have

$$
f \wedge g=f \otimes g-(-1)^{|f||g|} g \otimes f=-(-1)^{|f||g|} g \wedge f .
$$

Naturally a basis for NS is found by adding the central element $\gamma$ to the given basis of $k(1)$. The linear map $\varphi$ defined by

$$
\varphi\left(t^{p+1}\right)=2 L_{-p}, \quad \varphi\left(\theta t^{q}\right)=G_{1 / 2-q}, \quad \varphi(\gamma)=\frac{1}{4} D \quad(p, q \in \mathbb{Z})
$$


is an isomorphism that explicitly describes the connection between the description of the NeveuSchwarz algebra given in (1) and the one presented above. Besides the $\mathbb{Z}_{2}$ grading of $N S$,

$$
\left.N S=\underset{j \in Z_{2}}{\oplus} N S_{j}, \quad N S_{0}=\underset{j \in \mathbb{Z}}{\oplus}\left\langle e_{2 j}\right\rangle\right) \oplus\langle\gamma\rangle, \quad N S_{1}=\underset{j \in \mathbb{Z}}{\oplus}\left\langle e_{2 j-1}\right\rangle,
$$

which describes $N S$ as a superspace, the element $e_{0}$ defines an inner grading on $k(1)$ and $N S$ by means of its adjoint action. The basis elements are homogeneous with respect to this inner $\mathbb{Z}$ grading. We denote the eigenspace of $a d\left(e_{0}\right)$ corresponding to the eigenvalue $\lambda$ by $N S_{(\lambda)}$; then we have

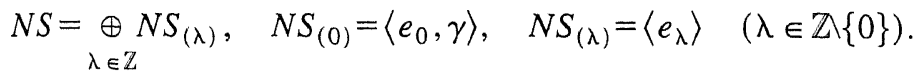

We remark that the inner grading is compatible with the $\mathbb{Z}_{2}$ grading in the sense that a homogeneous element of degree $\lambda$ has parity $\lambda$ modulo 2 . For later use we write out explicitly the commutators of the basis elements:

$$
\begin{gathered}
{\left[e_{2 j}, e_{2 k}\right]=2(k-j) e_{2 j+2 k}+2 k\left(k^{2}-1\right) \delta_{j+k, 0} \gamma,} \\
{\left[e_{2 j-1} e_{2 k}\right]=(k-2 j+1) e_{2 j+2 k-1},} \\
{\left[e_{2 j-1}, e_{2 k-1}\right]=e_{2 j+2 k-2}+2 k(k-1) \delta_{j+k, 1} \gamma,} \\
{\left[e_{j}, \gamma\right]=0 .}
\end{gathered}
$$

The commutators in $k(1)$ are the same with the omission of all terms involving the central element $\gamma$.

Theorem 1: The second cohomology group of $k(1)$ with coefficients in the adjoint representation is equal to zero:

$$
H^{2}(k(1) ; k(1))=0 \text {. }
$$

Proof: The $\mathbb{Z}$ grading of $k(1)$ induces a $\mathbb{Z}$ grading on the cochain complex $\left(C^{\bullet}(k(1) ; k(1)), d\right)$, so we can write

$$
C^{q}(k(1) ; k(1))=\prod_{\lambda \in \mathbb{Z}} C^{q}(k(1) ; k(1))_{(\lambda)} \quad(q \in \mathbb{Z}),
$$

and similarly for the corresponding cohomology groups. For details on the coboundary operator on this cochain complex and the corresponding cohomology groups we simply refer to Refs. 8, 13, and 14. Since nonzero cohomology classes can only exist at degree zero (see, e.g., Ref. 8 or 15), we know that

$$
H^{q}(k(1) ; k(1))_{(\lambda)}=0 \quad(q \in \mathbb{Z}),
$$

for all degrees $\lambda$ not equal to zero. This observation reduces the work to be done considerably; we can confine ourselves to studying homogeneous 2-cocycles of degree zero.

An arbitrary 2-cocycle of degree zero can be written as

$$
c\left(e_{j} \wedge e_{k}\right)=\alpha_{j, k} e_{j+k} \quad(j, k \in \mathbb{Z}),
$$

with coefficients $\alpha_{j, k} \in \mathrm{C}$ satisfying $\alpha_{j, k}=-(-1)^{j k} \alpha_{k, j}$. A cocycle represents a cohomology class, in general; it is, however, not unique since there exist other cohomologically equivalent cocycles. 
By application of coboundary corrections, we will determine a set of conditions for the coefficients $\alpha_{j, k}$ such that each cohomology class has a unique representative with coefficients that satisfy these specific conditions.

Lemma I: An arbitrary cohomology class of $H^{2}(k(1) ; k(1))_{0}$ can be represented by a 2 -cocycle as described by formula (9), with coefficients $\alpha_{j, k}$ satisfying

$$
\alpha_{-1,1}=\alpha_{0,1}=0, \quad \alpha_{j, k}=0 \quad(j k>0) .
$$

Proof: We perform coboundary corrections in several steps. We refer to each step by the condition it imposes on the coefficients $\alpha_{j, k}$.

Step 1: $\alpha_{j, k}=0 \quad\left(j, k \in \mathbb{Z}_{>0}\right)$

We define the Lie supersubalgebra of $k(1)$ spanned by the homogeneous elements of positive degree and denote it by

$$
k(1)_{+}=\underset{\lambda \in Z_{>0}}{\oplus} k(1)_{(\lambda)}
$$

In Ref. 7 we proved (by application of the Feigin-Fuchs spectral sequence; for its definition see Ref. 16) that $H^{2}\left(\mathrm{k}(1)_{+} ; \mathrm{k}(1)_{+}\right)_{(0)}=0$; hence the restriction of $c$ to $\wedge^{2}\left(k(1)_{+}\right)$is a coboundary. So there exists an element $b_{1}$ in $C^{1}\left(\mathrm{k}(1)_{+} ; \mathrm{k}(1)_{+}\right)_{(0)}$ such that $d\left(b_{1}\right)\left(e_{j} \wedge e_{k}\right)=c\left(e_{j} \wedge e_{k}\right)$ for all $j, k \in \mathbb{Z}_{>0}$. We expand $b_{1}$ trivially from $k(1)_{+}$to $k(1)$ by defining $b_{1}\left(e_{j}\right)=0$ for all $j \in \mathbb{Z}_{\leqslant 0}$ and we replace $c$ by $c^{\prime}=c-d\left(b_{1}\right)$. The 2-cocycle $c^{\prime}$ has coefficients $\alpha_{j, k}^{\prime}$ satisfying $\alpha_{j, k}^{\prime}=0$ for all $j, k \in \mathbb{Z}_{>0}$. From here on we will denote $c^{\prime}$ again by $c$.

Step 2: $\alpha_{j, k}=0 \quad\left(j, k \in \mathbb{Z}_{<0}\right)$

Similar to the previous step, we define

$$
k(1)_{-}=\underset{\lambda \in \mathbb{Z}_{<0}}{\oplus} k(1)_{(\lambda)}
$$

this is the Lie supersubalgebra of $k(1)$ spanned by the homogeneous elements of negative degree. It is isomorphic to $k(1)_{+}$by the isomorphism $\varphi: k(1)_{+} \rightarrow k(1)_{-}$, satisfying

$$
\left\{\begin{array}{l}
\varphi\left(e_{2 j}\right)=-e_{-2 j} \\
\varphi\left(e_{2 j-1}\right)=i e_{1-2 j}
\end{array} \quad\left(j \in \mathbb{Z}_{>0}\right) .\right.
$$

Hence, $H^{2}\left(k(1)_{-} ; k(1)_{-}\right)_{(0)}=0$ and there exists an element $b_{2}$ in $C^{1}(k(1) ; k(1))_{(0)}$ such that $\left(d\left(b_{2}\right)-c\right)\left(e_{j} \wedge e_{k}\right)=0, \quad\left(j, k \in \mathbb{Z}_{<0}\right), \quad$ and $b_{2}\left(e_{j}\right)=0, \quad(j \in \mathbb{N})$. In particular, we have $d\left(b_{2}\right)\left(e_{j} \wedge e_{k}\right)=0$ for all $j, k \in \mathbb{Z}_{>0}$, so $d\left(b_{2}\right)$ respects the previous conditions for the coefficients $\alpha_{j, k}$. We adjust $c$ to $c-d\left(b_{2}\right)$ and obtain $\alpha_{j, k}=0$ for all $j, k \in \mathbb{Z}_{<0}$.

Step 3: $\alpha_{0,1}=0$

We define the element $b_{3}$ in $C^{1}(k(1) ; k(1))_{(0)}$ by $b_{3}\left(e_{j}\right)=\delta_{j, 0} e_{0} \quad(j \in \mathbb{Z})$. The coboundary $d\left(b_{3}\right)$ satisfies $d\left(b_{3}\right)\left(e_{j} \wedge e_{k}\right)=0 \quad(j k>0)$, so it respects the preceding conditions for the coefficients $\alpha_{j, k}$. Since $d\left(b_{3}\right)\left(e_{0} \wedge e_{1}\right)=e_{1}$ we can adjust $c$ such that $\alpha_{0,1}$ becomes zero.

Step 4: $\alpha_{-1,1}=0$

Define $b_{4} \in C^{1}(k(1) ; k(1))_{(0)}$ by $b_{4}\left(e_{j}\right)=j e_{j} \quad\left(j \in \mathbb{Z}_{>0}\right)$ and $b_{4}\left(e_{j}\right)=0 \quad\left(j \in \mathbb{Z}_{\leqslant 0}\right)$. One easily verifies that $d\left(b_{4}\right)\left(e_{j} \wedge e_{k}\right)=0$ for $j k \geqslant 0$, hence the coboundary $d\left(b_{4}\right)$ respects the preceding conditions for $\alpha_{j, k}$. On account of $d\left(b_{4}\right)\left(e_{-1} \wedge e_{1}\right)=e_{0}$ it is possible to adjust $c$ such that $\alpha_{-1,1}=0$.

It is not yet clear that a representative of the form described in the previous lemma is unique. We will prove that any 2-cocycle that satisfies these conditions is equal to zero. The cocycle condition $d(c)=0$ gives rise to four types of equations for the coefficients $\alpha_{j, k}$. Since we will need them frequently we write down these equations explicitly: $d(c)\left(e_{2 j} \wedge e_{2 k} \wedge e_{2 l}\right)=0$ gives 


$$
\begin{aligned}
& (k-j) \alpha_{2 j+2 k, 2 l}-(l-j) \alpha_{2 j+2 l, 2 k}+(l-k) \alpha_{2 l+2 k, 2 j} \\
& \quad-(l+k-j) \alpha_{2 k, 3 l}+(j+l-k) \alpha_{2 j, 2 l}-(j+k-l) \alpha_{2 j, 2 k}=0 ;
\end{aligned}
$$

$d(c)\left(e_{2 j-1} \wedge e_{2 k} \wedge e_{2 l}\right)=0$ gives

$$
\begin{aligned}
& (k-2 j+1) \alpha_{2 j+2 k-1,2 l}-(l-2 j+1) \alpha_{2 j+2 l-1,2 k}+2(l-k) \alpha_{2 k+2 l, 2 j-1} \\
& \quad-(l+k-2 j+1) \alpha_{2 k, 2 l}+(2 l+2 j-k-1) \alpha_{2 j-1,2 l}-(2 j+2 k-l-1) \alpha_{2 j-1,2 k}=0 ;
\end{aligned}
$$

$d(c)\left(e_{2 j-1} \wedge e_{2 k-1} \wedge e_{2 l}\right)=0$ gives

$$
\begin{aligned}
& \alpha_{2 j+2 k-2,2 l}-(l-2 j+1) \alpha_{2 j+2 l-1,2 k-1}-(l-2 k+1) \alpha_{2 k+2 l-1,2 j-1} \\
& \quad-\alpha_{2 k-1,2 l}-\alpha_{2 j-1,2 l}-2(j+k-l-1) \alpha_{2 j-1,2 k-1}=0
\end{aligned}
$$

and $d(c)\left(e_{2 j-1} \wedge e_{2 k-1} \wedge e_{2 l-1}\right)=0$ gives

$$
\begin{gathered}
\alpha_{2 j+2 k-2,2 l-1}+\alpha_{2 j+2 l-2,2 k-1}+\alpha_{2 k+2 l-2,2 j-1}-(k+l-2 j) \alpha_{2 k-1,2 l-1} \\
-(j+l-2 k) \alpha_{2 j-1,2 l-1}-(j+k-2 l) \alpha_{2 j-1,2 k-1}=0 .
\end{gathered}
$$

By substituting specific values for $j, k$, and $l$ in these equations we prove in several steps that all coefficients $\alpha_{j, k}$ are equal to zero. Again we refer to each step by the condition it imposes on the coefficients.

Step 1: $\alpha_{0, p}=0(p \in \mathbb{Z})$

We substitute $(j, l)=(k, 0)$ in $(12)$ and find $\alpha_{4 k-2,0}=2 \alpha_{2 k-1,0}$. In particular $k=1$ yields $\alpha_{0,2}=0$, then by substituting $(j, k, l)=\left(0, \mathbb{Z}_{>0}, \mathbb{Z}_{>0}\right)$ in (10) we find $(l-k)\left(\alpha_{2 l+2 k, 0}-\alpha_{2 l, 0}-\alpha_{2 k, 0}\right)=0$ and $\alpha_{0,2 l}=l \alpha_{0,2}=0$ for all $l \in \mathbb{Z}_{>0}$. A similar proof can be given for $l \in \mathbb{Z}_{<0}$ since putting $(j, k, l)=(0,1,0)$ in (12) yields $\alpha_{-1,0}=0$.

Step 2: $\alpha_{-1, p}=\alpha_{1, p}=0 \quad(p \in \mathbb{Z})$

We already have $\alpha_{-1, p}=0$ for $p \leqslant 1$; in order to show that $\alpha_{-1, p}=0$ for $p \geqslant 2$ we take $(j, k, l)=\left(0,1, \mathbb{Z}_{>0}\right)$ and $(j, k, l)=\left(0, \mathbb{Z}_{>0}, 1\right)$ in $(12)$, yielding $-(l-1) \alpha_{-1,2 l+1}-\alpha_{-1,2 l}=0$ and $-2(1-k) \alpha_{-1,2 k+1}-\alpha_{-1,2}-2(k-2) \alpha_{-1,2 k-1}=0$. The choice $l=1$ in the first equation gives $\alpha_{-1,2}=0$, and by that the second one yields $\alpha_{-1,2 k+1}=(k-2) \alpha_{-1,2 k-1} /(k-1)$. The choice $k=2$ gives $\alpha_{-1,5}=0$ and by induction we find $\alpha_{-1,2 p+1}=0$ for $p \geqslant 2$. Then again, the first implies $\alpha_{-1, p}=0$ for all $p \geqslant 4$. The last case $\alpha_{-1,3}=0$ follows from substituting $(j, k, l)=(10,0,2)$ in (13). The case $\alpha_{1, p}=0$ for all $p \in \mathbb{Z}$ can be handled similarly.

Step 3: $\alpha_{2,2 p}=\alpha_{-2,2 p}=0(p \in \mathbb{Z})$

We consider $\alpha_{2,2 p}$ with $p \in \mathbb{Z}_{<0}$. Putting $(j, k, l)=(1,1,-1)$ in (12) yields $\alpha_{-2,2}=0$ and $(j, k, l)=\left(-1, \mathbb{Z}_{<0}, 1\right)$ in $(10)$ gives $(k+1) \alpha_{2 k-2,2}-(k+2) \alpha_{2 k, 2}=0$. So by taking $k=-2$ we obtain $\alpha_{2,-6}=0$ and inductively $\alpha_{2,2 p}=0$ for all $p \leqslant-3$. The condition $\alpha_{2,-4}=0$ follows from $(j, k, l)$ $=(-3,-2,1)$ in $(10)$. The case $\alpha_{-2,2 p}$ can be handled similarly.

Step 4: $\alpha_{4,2 p}=0(p \in \mathbb{Z})$

We only need to consider $p<-1$. We substitute $(j, k, l)=\left(-1, \mathbb{Z}_{<0}, 2\right)$ in $(10)$ and obtain $(k+1) \alpha_{2 k-2,4}-(k+3) \alpha_{2 k, 4}=0$. The choice $k=-3$ yields $\alpha_{4,2 p}=0$ for $p \leqslant-4$. By combining $k=-2$ with $(j, k, l)=(-3,-2,2)$ in $(10)$, we obtain $\alpha_{4,-6}=\alpha_{4,-4}=0$ and hence $\alpha_{4,2 p}=0$ for all $p$ in $\mathbb{Z}$.

Step 5: $\alpha_{2 n, 2 p}=0(n, p \in \mathbb{Z})$

We only need to consider $\alpha_{2 n, 2 p}$ with $n \in \mathbb{N}$ and $p \in \mathbb{Z}$. The cases $n=0,1,2$ are already evident. We proceed by induction, assuming that $\alpha_{2 j, 2 p}=0$ for all $j \leqslant n$. Substituting $(j, k, l)=(p, 1, n)$ in (10) gives $(n-1) \alpha_{2 n+2,2 p}=0$.

Step 6: $\alpha_{2, p}=0,(p \in \mathbb{Z})$

We only need to consider odd $p$. Put $(k, l)=(1,1)$ in $(13)$, then $\alpha_{2,2 j-1}=0$. 
Step 7: $\alpha_{3, p}=0(p \in \mathbb{Z})$

We put $(j, k)=(2,0)$ in (12) and (13) and find $\alpha_{2 l, 3}-(l+1) \alpha_{3,2 l-1}=0$ and $\alpha_{2 l-2,3}-(l+2) \alpha_{3,2 l-1}=0$. Setting $l=-2$ yields by induction $\alpha_{3, p}=0$ for $p \leqslant-6$. The case $p=-4$ follows from, e.g., $(j, k, l)=(2,-10,-12)$ in (11) and putting $l=-1$ and $l=-2$ in the equations above yield $\alpha_{3,-3}=\alpha_{3,-5}=0$. By this and previous steps all coefficients $\alpha_{3, p}$ are equal to zero.

Step 8: $\alpha_{n, p}=0(n, p \in \mathbb{Z})$

We only need to prove this for $n \in \mathbb{N}$ and $p \in \mathbb{Z}$. We use induction on $n$. The cases $n=0,1,2,3$ are evident on account of previous steps. It is completed by the substitutions

$$
\begin{gathered}
(j, k, l)=(1, p, n) \text { in }(11), \quad(1-n) \alpha_{2 n+1,2 p}=0 \quad(n \geqslant 2), \\
(j, k, l)=(p+1,1, n) \text { in }(12), \quad(1-n) \alpha_{2 n+1,2 p+1}=0 \quad(n \geqslant 2), \\
(j, k, l)=(p+1,1, n) \text { in }(13), \quad \alpha_{2 n, 2 p+1}=0 .
\end{gathered}
$$

This completes the proof that any 2-cocycle of degree zero is a coboundary. So by the previous remarks on the inner grading we have $H^{2}(k(1) ; k(1))=0$.

We will use the same technique to prove that the central extension $N S$ is rigid.

Theorem 2: The second cohomology group of NS with coefficients in the adjoint representation is equal to zero:

$$
H^{2}(N S ; N S)=0
$$

Proof: Due to the inner grading of $N S$ it suffices to prove that $H^{2}(N S ; N S)_{0}=0$. An arbitrary 2-cocycle of degree zero can be written as

$$
\begin{gathered}
c\left(e_{j} \wedge e_{k}\right)=\alpha_{j, k} e_{j+k}+\delta_{j+k, 0} \beta_{k} \gamma \quad(j, k \in \mathbb{Z}), \\
c\left(\gamma \wedge e_{j}\right)=\lambda_{j} e_{j}+\delta_{j, 0} \mu \gamma \quad(j \in \mathbb{Z}),
\end{gathered}
$$

where $\alpha_{j, k}, \beta_{k}, \lambda_{j}$, and $\mu$ are complex numbers satisfying

$$
\alpha_{j, k}=-(-1)^{j k} \alpha_{k, j} \quad(j, k \in \mathbb{Z}), \quad \beta_{-k}=-(-1)^{k} \beta_{k} \quad(k \in \mathbb{Z}) .
$$

Again we use coboundary corrections to choose representatives of a specific form. The notation will be similar to the one in Theorem 1 .

Lemma 2: An arbitrary cohomology class of $H^{2}(N S ; N S)_{0}$ can be represented by a 2-cocycle as described by formula (14), with coefficients satisfying

$$
\alpha_{-1,1}=\alpha_{0,1}=\beta_{1}=\beta_{3}=\lambda_{1}=0, \quad \alpha_{j, k}=0 \quad(j k>0) .
$$

Proof:

Step 1: $\alpha_{-1,1}=\alpha_{0,1}=0 \quad \alpha_{j, k}=0(j k>0)$

Use the coboundaries $d\left(b_{l}\right)(1 \leqslant l \leqslant 4)$, where $b_{l}$ is the extension of the element $b_{l}$ given in Theorem 1 , defined by $b_{l}(\gamma)=0$.

Step 2: $\lambda_{1}=0$

We define $b_{5}$ in $C^{1}(N S ; N S)_{(0)}$ by $b_{5}(\gamma)=e_{0}, b_{5}\left(e_{j}\right)=0,(j \in \mathbb{Z})$; then $d\left(b_{5}\right)\left(\gamma \wedge e_{1}\right)=e_{1}$ so we obtain $\lambda_{1}=0$.

Step 3: $\beta_{1}=0$

We define $b_{6}$ in $C^{1}(N S ; N S)_{(0)}$ by $b_{6}(\gamma)=0, b_{6}\left(e_{l}\right)=\delta_{l, 0} \gamma,(l \in \mathbb{Z})$, and we see that $d\left(b_{6}\right)\left(e_{-1} \wedge e_{1}\right)=-\gamma$. By this $\beta_{1}$ becomes zero.

Step 4: $\beta_{3}=0$ 
Define $b_{7}$ in $C^{1}(N S ; N S)_{(0)}$ by $b_{7}(\gamma)=\gamma, b_{7}\left(e_{l}\right)=0,(l \in \mathbb{Z})$; then $d\left(b_{7}\right)\left(e_{-3} \wedge e_{3}\right)=-4 \gamma$; hence $\beta_{3}$ becomes zero.

It can easily be verified that all these corrections respect the preceding ones.

We will prove that any representative $c$ of this form is equal to zero. For that purpose we write out some consequences of the cocycle condition $d(c)=0$ :

$$
\begin{aligned}
d(c)\left(\gamma \wedge e_{2 j-1} \wedge e_{2 k-1}\right)= & \left(\lambda_{2 j+2 k-2}-\lambda_{2 k-1}-\lambda_{2 j-1}\right) e_{2 j+2 k-2} \\
& +\delta_{j+k, 1}\left(\mu-\lambda_{2 j-1} 2 j(j-1)-\lambda_{2 k-1} 2 k(k-1)\right) \gamma=0 \\
d(c)\left(\gamma \wedge e_{2 j-1} \wedge e_{2 k}\right)= & (k-2 j+1)\left(\lambda_{2 j+2 k-1}-\lambda_{2 k}-\lambda_{2 j-1}\right) e_{2 j+2 k-1}=0 \\
d(c)\left(\gamma \wedge e_{2 j} \wedge e_{2 k}\right)= & 2(k-j)\left(\lambda_{2 j+2 k}-\lambda_{2 j}-\lambda_{2 k}\right) e_{2 j+2 k} \\
& +2 k \delta_{j+k, 0}\left(2 \mu-\left(k^{2}-1\right)\left(\lambda_{2 j}+\lambda_{2 k}\right)\right) \gamma=0 .
\end{aligned}
$$

We take $(j, k)=(1,0)$ in (15) and $k=0$ in (16) and obtain $\mu=\lambda_{0}=0$. By substituting $j=1$ in (15) and (16), we find $\lambda_{k}=\lambda_{1}=0$ for all $k \in \mathbb{Z}$. Since the equations (10)-(13) still hold, we conclude that $\alpha_{j, k}=0$ for all $j$ and $k$ in $\mathbb{Z}$. Finally, we prove that the coefficients $\beta_{j}$ are equal to zero,

$$
\begin{gathered}
d(c)\left(e_{2 j-1} \wedge e_{2 k-1} \wedge e_{2-2 j-2 k}\right) \\
=-\left\{\beta_{2-2 j-2 k}+(3 j+k-2) \beta_{2 k-1}+(3 k+j-2) \beta_{2 j-1}\right\} \gamma=0, \\
d(c)\left(e_{2 j} \wedge e_{2 k} \wedge e_{-2 j-2 k}\right)=2\left\{(k-j) \beta_{2 j+2 k}-(2 j+k) \beta_{2 k}+(2 k+j) \beta_{2 j}\right\} \gamma=0 .
\end{gathered}
$$

We substitute $(j, k)=(1,1)$ and $(j, k)=(1,2)$ in $(18)$ and obtain $\beta_{2}=\beta_{4}=0$. The choice $j=1$ in (19) yields $\beta_{2 k+2}=[(k+2) /(k-1)] \beta_{2 k} \quad(k \neq 1)$, and by induction all $\beta_{2 k}$ are equal to zero. Then $j=k$ in (18) implies $\beta_{2 k-1}=0$ for all $k$ in $\mathbb{Z}$ and the proof is complete.

\section{THE RIGIDITY OF $\boldsymbol{k}^{+}(1)$ AND $R$}

The Lie superalgebra $k^{+}(1)$ (see Ref. 9) looks quite similar to $k(1)$; it consists of the $\mathbb{Z}_{2}$-graded vector space $\mathrm{C}\left[t, t^{-1}, \theta \sqrt{t}\right]$ equipped with the commutator of formula (3). We define a basis of $k^{+}(1)$ by $\left\{e_{p}\right\}_{p \in Z}$, where

$$
e_{2 p}=t^{p+1}, \quad e_{2 p-1}=\theta t^{p} \sqrt{t} \quad(p \in \mathbb{Z}) .
$$

The elements in this basis are homogeneous; the parity of $e_{p}$ is $p$ modulo 2 . The Ramond algebra is the unique central extension of $k^{+}(1)$. It is described by the 2-cocycle of formula (5) and we shall denote it by $R$. Let $\gamma$ denote the central element of $R$. An isomorphism that relates this description with the one presented by formula (2) in the Introduction can be given by

$$
\varphi\left(t^{p+1}\right)=2 L_{-p}+\frac{1}{16} \delta_{p, 0} D, \quad \varphi\left(\theta t^{q} \sqrt{t}\right)=F_{-q}, \quad \varphi(\gamma)=\frac{1}{4} D \quad(p, q \in \mathbb{Z}) .
$$

We will use the inner grading of $R$ corresponding to the special element $\frac{1}{2} e_{0}$. This boils down to

$$
R=\underset{\lambda \in \mathbb{Z}}{\oplus R_{(\lambda)}, \quad R_{(\lambda)}}=\left\langle e_{2 l-1}, e_{2 \lambda}\right\rangle \quad(\lambda \in \mathbb{Z}\{0\}), \quad R_{(0)}=\left\langle e_{-1}, e_{0}, \gamma\right\rangle
$$

The basis elements are homogeneous with respect to this $\mathbb{Z}$ grading, however, the $\mathbb{Z}_{2}$ grading of $R$ is not compatible with this $\mathbb{Z}$ grading. To be more precise, we have

$$
R_{(\lambda)}=R_{(\lambda), 0} \oplus R_{(\lambda), 1}=\left\langle e_{2 \lambda}\right\rangle \oplus\left\langle e_{2 \lambda-1}\right\rangle \quad(\lambda \neq 0)
$$


and

$$
R_{(0)}=R_{(0), 0} \oplus R_{(0), 1}=\left\langle e_{0}, \gamma\right\rangle \oplus\left\langle e_{-1}\right\rangle .
$$

This makes the rigidity proof in this case slightly more technical than the one presented in the previous section. For later use we write out the commutators of the basis elements:

$$
\begin{gathered}
{\left[e_{2 j}, e_{2 k}\right]=2(k-j) e_{2 j+2 k}+2 k\left(k^{2}-1\right) \delta_{j+k, 0} \gamma,} \\
{\left[e_{2 j-1}, e_{2 k}\right]=(k-2 j) e_{2 j+2 k-1},} \\
{\left[e_{2 j-1}, e_{2 k-1}\right]=e_{2 j+2 k}+2\left(k^{2}-\frac{1}{4}\right) \delta_{j+k, 0} \gamma,} \\
{\left[e_{j}, \gamma\right]=0 .}
\end{gathered}
$$

The commutators in $k^{+}(1)$ are as described above with the omission of all the terms involving the central element $\gamma$.

Theorem 3: The even part of the second cohomology group of $k^{+}(1)$ with coefficients in the adjoint representation is equal to zero:

$$
H^{2}\left(k^{+}(1) ; k^{+}(1)\right)=0 \text {. }
$$

Proof: Due to the inner grading of $k^{+}(1)$, we only need to prove $H^{2}\left(k^{+}(1) ; k^{+}(1)\right)_{(0), 0}=0$, i.e. we can restrict ourselves to studying even 2-cocycles of degree zero. We will use the same technique as described in the two proofs of the previous section, therefore we will confine ourselves to shortly indicating the various steps without discussing them in detail.

An even 2-cocycle $c$ of degree zero is of the following form:

$$
\begin{gathered}
c\left(e_{2 j} \wedge e_{2 k}\right)=\alpha_{2 j, 2 k} e_{2 j+2 k}, \\
c\left(e_{2 j-1} \wedge e_{2 k}\right)=\alpha_{2 j-1,2 k} e_{2 j+2 k-1}, \\
c\left(e_{2 j-1} \wedge e_{2 k-1}\right)=\alpha_{2 j-1,2 k-1} e_{2 j+2 k},
\end{gathered}
$$

with $\alpha_{j, k} \in \mathbb{C}$ and $\alpha_{j, k}=-(-1)^{j k} \alpha_{k, j}(j, k \in \mathbb{Z})$.

Lemma 3: An arbitrary cohomology class of $\mathrm{H}^{2}\left(k^{+}(1) ; k^{+}(1)\right)_{(0), 0}$ can be represented by a 2 -cocycle as described by formula (22), with coefficients $\alpha_{j, k}$ satisfying

$$
\alpha_{-2,-1}=\alpha_{-2,1}=\alpha_{0,1}=0, \quad \alpha_{j, k}=0 \quad(j, k>0 ; j, k<-1) .
$$

Proof:

Step 1: $\alpha_{j, k}=0(j, k>0)$

Define

$$
k^{+}(1)_{+}=\underset{\lambda \in Z_{>0}}{\oplus} k^{+}(1)_{(\lambda)}
$$

this is the Lie supersubalgebra of $k^{+}(1)$ spanned by the homogeneous elements of positive degree. From Ref. 7 we know that $H^{2}\left(k^{+}(1)_{+} ; k^{+}(1)_{+}\right)_{(0)}=0$; hence the restriction of $c$ to $\wedge^{2}\left(k^{+}(1)_{+}\right)$is a coboundary. By subtracting $d\left(b_{1}\right)$ from $c$, where $b_{1}$ denotes the trivial expansion of the element that yields the coboundary mentioned above, we obtain a 2-cocycle with coefficients $\alpha_{j, k}$ satisfying the described property.

Step 2: $\alpha_{j, k}=0(j, k<-1)$

Define 


$$
k^{+}(1)_{-}=\underset{\lambda \in \mathbb{Z}_{<0}}{\oplus} k^{+}(1)_{(\lambda)}
$$

and note that $k^{+}(1)_{-}$is isomorphic to $k^{+}(1)_{+}$by the isomorphism $\varphi: k^{+}(1)_{+} \rightarrow k^{+}(1)_{-}$, given by

$$
\left\{\begin{array}{l}
\varphi\left(e_{2 j}\right)=-e_{-2 j} \\
\varphi\left(e_{2 j-1}\right)=i e_{-2 j-1}
\end{array} \quad\left(j \in \mathbb{Z}_{>0}\right) .\right.
$$

By a similar argument as given in Step 1 , we obtain $\alpha_{j, k}=0$ for $j, k<-1$.

Step 3: $\alpha_{0,1}=0$

Use $d\left(b_{2}\right)$ with $b_{2}\left(e_{j}\right)=\delta_{j, 0} e_{0}(j \in \mathbb{Z})$; then $d\left(b_{2}\right)\left(e_{0} \wedge e_{1}\right)=2 e_{1}$.

Step 4: $\alpha_{-2,-1}=0$

Use $d\left(b_{3}\right)$ with $b_{3}\left(e_{j}\right)=\delta_{j+1,0} e_{-1}(j \in \mathbb{Z}) ;$ then $d\left(b_{3}\right)\left(e_{-2} \wedge e_{-1}\right)=e_{-3}$.

Step 5: $\alpha_{-2,1}=0$

Define $b_{4}$ by $b_{4}\left(e_{2 j}\right)=j e_{2 j}, b_{4}\left(e_{2 j-1}\right)=j e_{2 j-1} \quad\left(j \in \mathbb{Z}_{\leqslant 0}\right)$, and $b_{4}\left(e_{j}\right)=0 \quad\left(j \in \mathbb{Z}_{>0}\right) ;$ then $d\left(b_{4}\right)\left(e_{-2} \wedge e_{1}\right)=-3 e_{-1}$.

One can easily verify that each coboundary respects the conditions that have been imposed on the coefficients $\alpha_{j, k}$ in preceding steps.

By similar inductive arguments, as demonstrated in the preceding theorems, one can prove that any 2-cocycle of the form described in the lemma is equal to zero. By that the proof is complete.

Theorem 4: The even part of the second cohomology group of $R$ with coefficients in the adjoint representation is equal to zero:

$$
H^{2}(R ; R)_{0}=0
$$

Proof: Due to the inner grading of $R$, it is sufficient to prove that $H^{2}(R ; R)_{(0), 0}=0$. An arbitrary even 2-cocycle of degree zero can be written as

$$
\begin{gathered}
c\left(e_{2 j} \wedge e_{2 k}\right)=\alpha_{2 j, 2 k} e_{2 j+2 k}+\delta_{j+k, 0} \beta_{2 k} \gamma, \\
c\left(e_{2 j-1} \wedge e_{2 k}\right)=\alpha_{2 j-1,2 k} e_{2 j+2 k-1}, \\
c\left(e_{2 j-1} \wedge e_{2 k-1}\right)=\alpha_{2 j-1,2 k-1} e_{2 j+2 k}+\delta_{j+k, 0} \beta_{2 k-1} \gamma, \\
c\left(c \gamma \wedge e_{j}\right)=\lambda_{j} e_{j}+\delta_{j, 0} \mu \gamma,
\end{gathered}
$$

where $\alpha_{j, k}, \beta_{k}, \lambda_{j}$, and $\mu$ are complex numbers such that

$$
\alpha_{j, k}=-(-1)^{j k} \alpha_{k, j} \quad(j, k \in \mathbb{Z}), \quad \beta_{-2 k}=-\beta_{2 k}, \quad \beta_{2 k-1}=\beta_{-2 k-1} \quad(k \in \mathbb{Z}) .
$$

Lemma 4: An arbitrary cohomology class of $H^{2}(R ; R)_{(0), 0}$ can be represented by a 2-cocycle as described by formula (23), with coefficients $\alpha_{j, k}$ satisfying

$$
\alpha_{-2,-1}=\alpha_{-2,1}=\alpha_{0,1}=0, \quad \alpha_{j, k}=0 \quad(j, k>0 ; j, k<-1),
$$

and coefficients $\beta_{k}$ and $\lambda_{j}$ that satisfy

$$
\beta_{1}=\beta_{2}=\lambda_{2}=0 \text {. }
$$

Proof: It is evident that the restrictions from Step 1-5 from Theorem 3 can also be obtained for $\alpha_{j, k}$ in this case. Furthermore, we perform the following steps.

Step 1: $\beta_{2}=0$

Use $d\left(b_{1}\right)$ with $b_{1}\left(e_{j}\right)=\delta_{j, 0} \gamma(j \in \mathbb{Z})$, and $b_{1}(\gamma)=0$; then $d\left(b_{1}\right)\left(e_{-2} \wedge e_{2}\right)=-4 \gamma$. 
Step 2: $\beta_{1}=0$

Use $d\left(b_{2}\right)$ with $b_{2}(\gamma)=\gamma$ and $b_{2}\left(e_{j}\right)=0(j \in \mathbb{Z})$; then $d\left(b_{2}\right)\left(e_{-3} \wedge e_{1}\right)=-\frac{3}{2} \gamma$.

Step 3: $\lambda_{2}=0$

Use $d\left(b_{3}\right)$ with $b_{3}(\gamma)=e_{0}$ and $b_{3}\left(e_{j}\right)=0(j \in \mathbb{Z})$; then $d\left(b_{3}\right)\left(\gamma \wedge e_{2}\right)=2 e_{2}$.

We will prove that any 2-cocycle $c$ given by (23), with coefficients satisfying the conditions of the preceding lemma, is equal to zero,

$$
\begin{gathered}
d(c)\left(\gamma \wedge e_{2 j} \wedge e_{2 k}\right)=2(k-j)\left(\lambda_{2 j+2 k}-\lambda_{2 j}-\lambda_{2 k}\right) e_{2 j+2 k} \\
+\delta_{j+k, 0} 2 k\left(2 \mu-\left(k^{2}-1\right)\left(\lambda_{2 j}+\lambda_{2 k}\right)\right) \gamma=0, \\
d(c)\left(\gamma \wedge e_{2 j-1} \wedge e_{2 k}\right)=(k-2 j)\left(\lambda_{2 j+2 k-1}-\lambda_{2 j-1}-\lambda_{2 k}\right) e_{2 j+2 k-1}=0, \\
d(c)\left(\gamma \wedge e_{2 j-1} \wedge e_{2 j-1}\right)=\left(\lambda_{4 j}-2 \lambda_{2 j-1}\right) e_{4 j}+\delta_{j, 0}\left(\mu+\lambda_{-1}\right) \gamma=0 .
\end{gathered}
$$

From the substitution $(j, k)=(-1,1)$ in (24), we obtain $\mu=0$. Then $j=0$ in (26) yields $\lambda_{-1}=0$. From $k=1$ in (25) we conclude $\lambda_{2 j-1}=\lambda_{-1}=0$ for all $j \in \mathbb{Z}$. Finally, Eq. (25) implies $\lambda_{2 k}=0$ for all $k \in \mathbb{Z}$.

Since the conditions for the coefficients $\alpha_{j, k}$ from Lemma 4 coincide with the conditions in Lemma 3, the proof of the preceding theorem implies that all coefficients $\alpha_{j, k}$ are equal to zero. In order to show that $\beta_{k}$ equals zero for all $k \in \mathbb{Z}$, we write out the following two expressions:

$$
\begin{aligned}
& d(c)\left(e_{2 j} \wedge e_{2 k} \wedge e_{-2 j-2 k}\right)=2\left\{(k-j) \beta_{2 j+2 k}-(k+2 j) \beta_{2 k}+(2 k+j) \beta_{2 j}\right\} \gamma=0, \\
& d(c)\left(e_{2 j-1} \wedge e_{2 k-1} \wedge e_{-2 j-2 k}\right)=\left\{\beta_{2 j+2 k}-(k+3 j) \beta_{2 k-1}-(j+3 k) \beta_{2 j-1}\right\} \gamma=0 .
\end{aligned}
$$

We take $(j, k)=(1,1)$ in $(28)$ and obtain $\beta_{4}=0$. Then by inductive use of Eq. (27) we conclude that $\beta_{2 k}=0$ for all $k \in \mathbb{Z}$. Finally, $j=1$ in (28) gives $\beta_{2 k-1}=0$ for all $k \in \mathbb{Z}$. This completes the proof that any even 2-cocycle of degree zero is a coboundary.

\section{SOME FINAL REMARKS}

The even parts of the Lie superalgebras $N S$ and $R$ are isomorphic to the Virasoro algebra. Therefore one could consider the rigidity proofs of these superalgebras, as extensions of a rigidity proof for the Virasoro algebra. In fact, this is how the presented technique has arisen. This technique can also be used with respect to $W$ algebras, work on this is in progress.

In the introductory part of this paper we mentioned the possibility of deforming Lie superalgebras. A deformation of a Lie superalgebra $L$ is defined by a new commutator on $L$, which is a power series in a formal parameter, with the zeroth-order term equal to the bracket of $L$. Since the parameter involved is usually even, it is only the even part of the cohomology group of $L$ that is of interest with respect to the rigidity of $L$. Nevertheless, one can formally carry out the construction of a deformation of $L$ by using an odd parameter, such deformations are therefore called odd deformations. For details on this we refer to Ref. 8. On account of $\mathbb{Z}$ homogeneity; the cocycle that describes an odd deformation needs to be odd. By consequence, a sufficient condition for the rigidity of a Lie superalgebra with respect to these kinds of odd deformations is that the odd component of its second cohomology group with coefficients in the adjoint module is equal to zero. From Theorems 1 and 2 we learn that $k(1)$ and $N S$ satisfy this condition. We can give proofs similar to the ones given in Theorems 3 and 4 , to show that $H^{2}\left(k^{+}(1) ; k^{+}(1)\right)_{1}=0$ and $H^{2}(R ; R)_{1}=0$. Hence, we can conclude that the Lie superalgebras $k(1), k^{+}(1), N S$, and $R$ are also rigid with respect to odd deformations. 


\section{ACKNOWLEDGMENT}

Y.Y.K. supported in part by NWO Grant No. 07-30-001.

${ }^{1}$ M. Gerstenhaber, "On the deformations of rings and algebras," Ann. Math. 79, 59 (1964).

${ }^{2}$ A. Fialowski, "Deformations of nilpotent Kac-Moody algebras," Stud. Sci. Math. Hungary 19. 465 (1984).

${ }^{3}$ A. Fialowski, "On the deformations of $L_{1}$," Stud. Sci. Math. Hungary 20, 43.3 (1985)

${ }^{4}$ A. Fialowski, "Deformations of some infinite-dimensional Lie algebras," J. Math. Phys. 31, 1340 (1990).

${ }^{5} \mathrm{Yu}$. Yu. Kochetkov and G. F. Post, "Deformations of the nilpotent infinite-dimensional Lie algebra $L_{2}$," Funct Appl. 26, 304 (1992).

${ }^{6}$ N. van den Hijligenberg, Yu. Yu. Kochetkov, and G. F. Post, "Deformations of vector fields and Hamiltonian fields on the plane," Math. Comput. 64, 1215 (1995).

${ }^{7}$ N. van den Hijligenberg, "Deformations of nilpotent parts of the Neveu-Schwarz and Ramond superalgebras," J Phys. 35, 1427 (1994).

${ }^{8} \mathrm{~N}$. van den Hijligenberg, "Computations and applications of Lie superalgebra cohomology," Thesis, Univer Twente, Enschede, 1993.

${ }^{9}$ B. L. Feigin and D. A. Leites, "New Lie superalgebras of string theories," in Group Theoretical Methods in (Moscow, Nauka, 1983).

${ }^{10}$ A. Neveu and J. H. Schwarz, "Factorizable dual model pions," Nucl. Phys. B 31, 86 11971).

"P. Ramond, "Dual theory for free fermions," Phys. Rev. D 3, 2415 (1972).

${ }^{12}$ M. B. Green, J. H. Schwarz, and E. Witten, Superstring Theory (Cambridge University, (ambridge. 1987), p. 2

${ }^{13}$ D. A. Leites, "Cohomology of Lie superalgebras," Funct. Anal. Appl. 9. 75 (1975).

${ }^{14}$ K. C. Tripathy and M. K. Patra, "Cohomology theory and deformations of $2_{2}$-graded Lie algebras," J. Math. Pf 2822 (1990).

${ }^{15}$ D. B. Fuchs, Cohomology of Infinite-Dimensional Lie Algebras (Plenum, New York, 19861.

${ }^{16}$ B. L. Feigin and D. B. Fuchs, "Homology of the Lie algebra of vector tields on the line," Funct. Anal. Appl. (1980). 